\title{
Colonoscopic Finding of Patients with Lower Gastrointestinal Bleeding at Different Age Group in Eastern Part of India - An Observational Study
}

\section{Jayanta Paul}

Department of Gastroenterology, Desun Hospital and Heart Institute, Kolkata, India Received November 9, 2019; Accepted February 17, 2020.

Key words: Lower Gl bleeding - Eastern part of India - Etiologies - Hemorrhoids - Colon carcinoma - Anal fissure - Isolated rectal ulcer - Pancolitis - Colonoscopic findings

\begin{abstract}
Incidence of lower gastrointestinal (GI) bleeding (LGIB) is increasing over time. It can be seen in all age group patients, commonly associated with pre-existing comorbidities and is one of the common indications of colonoscopy. This study was done to identify common causes of LGIB in eastern part of India, because there is no previous study from Eastern India to identify the common causes of lower $\mathrm{Gl}$ bleeding diagnosed by colonoscopy in different age group patients. Consecutive 64 patients with LGIB were included in this study from June 2018 to March 2019. We divided our study population into three groups, such as group A (20 years to 40 years), group $B$ (41 years to 60 years), and group $C$ (more than 60 years). Data were entered into Excel and then transferred into SPSS version 22 for statistical analysis. Mean age of study population was $49.83 \pm 19.06$ years. Normal colonoscopic finding was seen in 7 patients (10.9\%). Most common colonoscopic findings of our study population were hemorrhoids $(n=32 ; 50 \%)$, anal fissure $(n=11$; $17.2 \%)$ and isolated rectal ulcer $(n=9 ; 14.1 \%)$. Colorectal growth was seen in 6 patients (9.4\%), among them female patients were more commonly affected than male patients. Therefore, most common causes of LGIB in eastern part of India are hemorrhoids, anal fissure and isolated rectal ulcer. Male individuals are more commonly affected by LGIB.
\end{abstract}

Mailing Address: Jayanta Paul, MD., MBBS, DNB, C/o Jitendra Chandra Paul, J+B Lodge, Santosh Sarani, Banamali Pur, Barasat, Kolkata 700124, India; Phone: (+91) 83489840 88; e-mail: dr.jayantapaul@gmail.com 


\section{Introduction}

Lower gastrointestinal bleeding (LGIB) is gastrointestinal $(\mathrm{Gl})$ bleeding originated from a source distal to the ligament of Treitz and is commonly presented with hematochezia which is different from the clinical presentation of upper Gl bleeding, which includes hematemesis and/or melena depending on the volume of bleeding and the speed of colonic transit. Approximately $85 \%$ of LGIB is from colon, $10 \%$ from bleeds are actually from upper gastrointestinal tract and present as hematochezia and 3-5\% from small intestines (Dutta and Panda, 2008). Acute LGIB is arbitrarily defined as bleeding of less than three days duration leading to instability of vital signs, anemia, and/or need for blood transfusion, and chronic LGIB is defined as slow blood loss over a period of several days. Incidence of LGIB in the western countries ranges from 20.5 to 27 cases/100,000 adults. In comparison with the western countries, in India, LGIB patients are younger, mortality rate is lower and re-bleed rate is 4\% (Farrell and Friedman, 2005). Lower Gl bleeding has an annual incidence of hospitalization of approximately $36 / 100,000$ population (Ghassemi and Jensen, 2013) and the colonoscopy is a primary method of investigation in presence of bleeding from lower Gl tract (Dar et al., 2015; Oakland et al., 2019).

The etiology and the epidemiology of LGIB depend on the life style, dietary habits, smoking, history of drug intake, age, longevity of the population, etc. The most common cause of LGIB in UK is diverticular bleeding and the second most frequent diagnoses are hemorrhoids, fissures and rectal ulcers (Oakland et al., 2019). Diverticular disease is the most common cause of LGIB in Brazil, followed by polyps, malignancy, inflammatory bowel disease and angiodysplasia (de Souza e Benevides and dos Santos, 2016).

In Asia, however, colon diverticulosis is not common and is a much less common cause of LGIB. In the Indian experience, the etiology differs significantly. Growth/ polyp are the most common colonoscopic finding in Jammu and Kashmir, India followed by inflammatory bowel lesions (Dar et al., 2015). An internal hemorrhoid is the most common cause of LGIB followed by ulcerative colitis in South India (Badiger et al., 2017). A study from USA showed that divericular bleeding (37\%) was the most common cause of severe LGIB followed by ischemic colitis (13.2\%), delayed post polypectomy induced bleeding (11.1\%), rectal ulcer (8.9\%), internal hemorrhoids (6.4\%) and colon angiomas (6.4\%) (Camus et al., 2017). Another study from western country discovered that the most common causes of LGIB were diverticulosis and ischemic colitis (Diamantopoulou et al., 2017). Study from Middle East indicated that the most common colonoscopic findings of LGIB were hemorrhoids followed by diverticulosis, neoplasm, rectal ulcer, colitis and polyps respectively, and colon was normal in 14.6\% patients (Alruzug et al., 2016).

There this is no previous study from Eastern India to find out the common causes of lower Gl bleeding in different age group patients diagnosed by colonoscopy. Aim of this study is to fill up this gap. 


\section{Material and Methods}

Colonoscopies in patients with lower gastrointestinal bleeding were prospectively evaluated from June 2018 to March 2019 in the endoscopy unit of Divine Nursing Home, Kolkata, India. This study included 64 patients aged 18 years or over, who presented with hematochezia, melena with normal upper GI Cendoscopy. Patients with poor bowel preparation and incomplete examination were excluded. All patients were advised to take liquid diet at dinner along with two $10 \mathrm{mg}$ of bisacodyl tablets on the day before procedure, while fasting over midnight. The medication used for bowel preparation in this study was two bottles of coloprep solution (each bottle contains magnesium sulphate $3.13 \mathrm{~g}+$ potassium $1.6 \mathrm{~g}+$ sodium chloride $17.5 \mathrm{~g}$ in $177 \mathrm{ml}$ of solution). $177 \mathrm{ml}$ of each bottle of coloprep solution was mixed with $573 \mathrm{ml}$ of drinking water to make it $750 \mathrm{ml}$. The Boston bowel preparation scale (BBPS) was used as bowel cleanliness rating scale. When required, tissue from the colonic lesion was sent for histopathological diagnosis. The following variables were studied: gender, age, coloscopic diagnosis, histopathologic diagnosis, site of the lesion. All colonoscopies were performed by using Olympus colonoscope. We divided our study population into three groups, such as group $A$ (20 years to 40 years), group B (41 years to 60 years), group C (more than 60 years). Data were entered into Excel and then transferred into SPSS version 22 for statistical analysis. Continuous value is expressed in the form of means $\pm S D$, while categorical data is expressed in the form of count and percent.

\section{Results}

Out of 64 patients with LGIB, 42 (65.6\%) were male and 22 (34.4\%) were female. Mean age of this study population was $49.83 \pm 19.06$ years. Normal colonoscopic finding was seen in 7 patients (10.9\%). Most common findings of our study population were hemorrhoids ( $n=32 ; 50 \%)$ (Figure 1$)$, anal fissure $(n=11 ; 17.2 \%)$ (Figure 2) and isolated rectal ulcer ( $n=9 ; 14.1 \%)$ (Figure 3). Anal fissure $(18.2 \%$ vs. $11.9 \%)$ and rectal ulcer ( $9.1 \%$ vs. $4.8 \%$ ) were more commonly seen in female patients than male patients. Colorectal growth (Figure 4) was seen in 6 patients (9.4\%), among them female patients were more commonly affected than male patients ( $9 \%$ vs. $4.8 \%$ ) (Table 1, Figure 5$)$.

In our study, maximum number of patients $(n=23)$ were in group $C$ (more than 60 years) and group A (20 to 40 years). In group C, common causes of LGIB were hemorrhoid $(n=13 ; 56.5 \%)$, rectal ulcer $(n=5 ; 21.7 \%)$, telangiectasia $(n=3 ; 13 \%)$ (Figure 6), diverticulae $(n=2 ; 8.7 \%)$, anal fissure $(n=2 ; 8.7 \%)$, colorectal growth $(n=1 ; 4.3 \%)$ and pancolitis $(n=1 ; 4.3 \%)$ (Figure 7$)$.

In group B (41 to 60 years), among 18 patients, 12 (66.7\%) and 6 (33.3\%) were male and female respectively. Most common cause of LGIB in group B was hemorrhoids $(n=9 ; 50 \%)$ and other causes were anal fissure $(n=4 ; 22.2 \%)$, colorectal growth $(n=3 ; 16.7 \%)$, rectal ulcer $(n=1 ; 5.6 \%)$, pancolitis $(n=1 ; 5.6 \%)$. Normal colonoscopic finding was in 4 patients (22.2\%) in group B (Figure 8). 


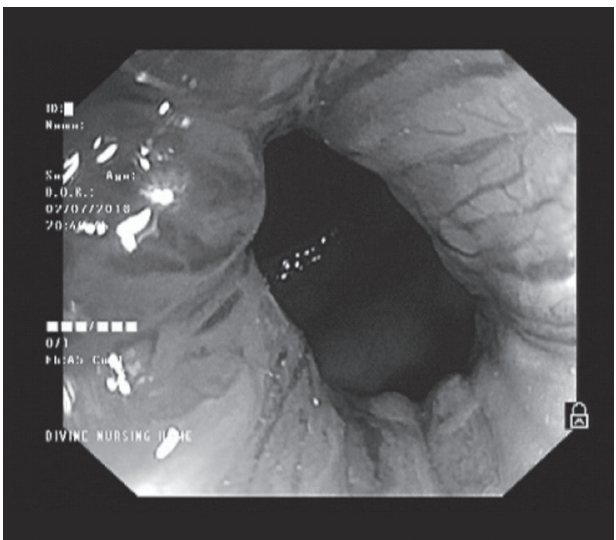

Figure 1 - Hemorrhoids.

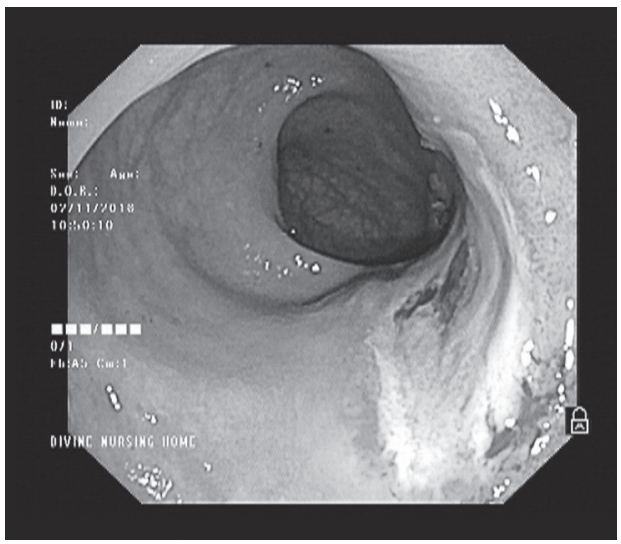

Figure 3 - Isolated rectal ulcer.

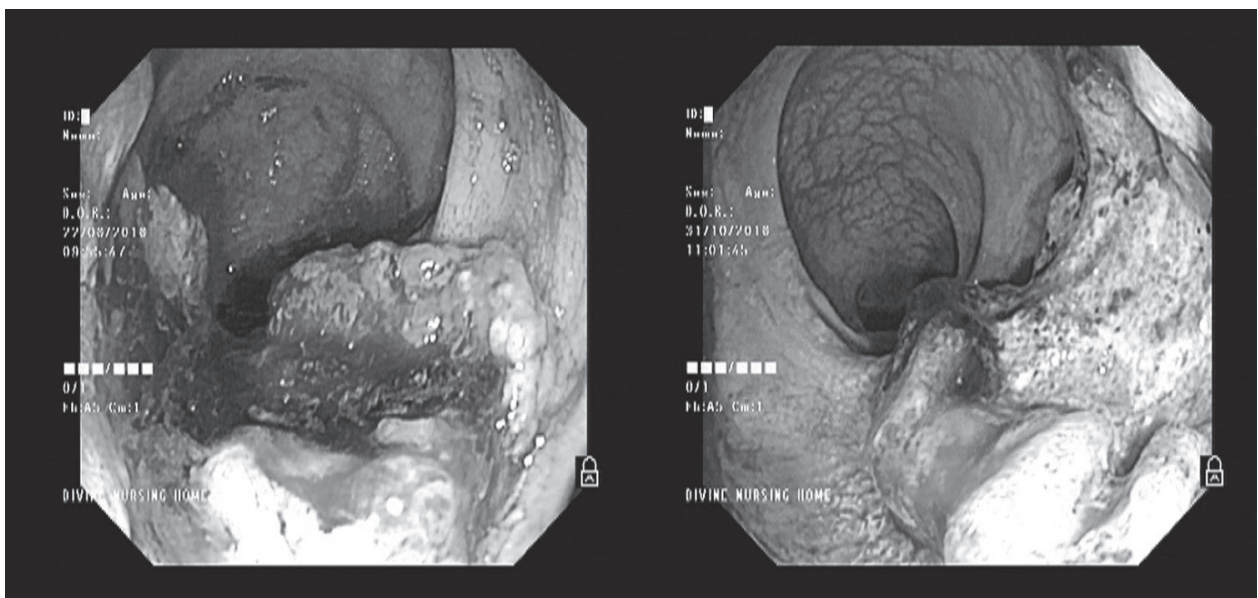

Figure 4 - Rectal growth.

Paul J.

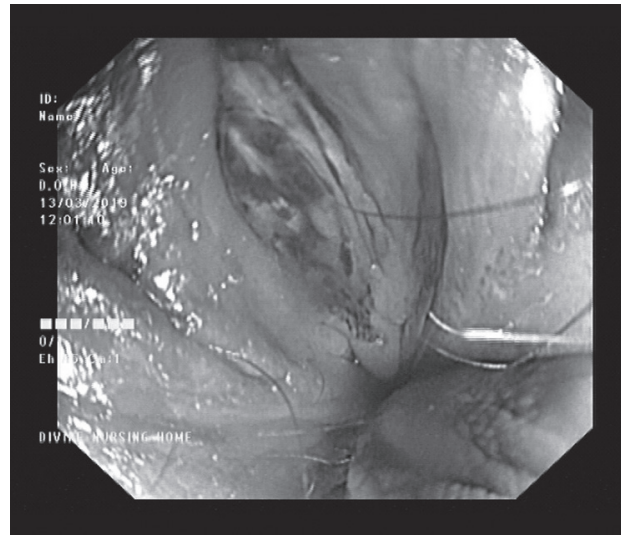

Figure 2 - Anal fissure. 


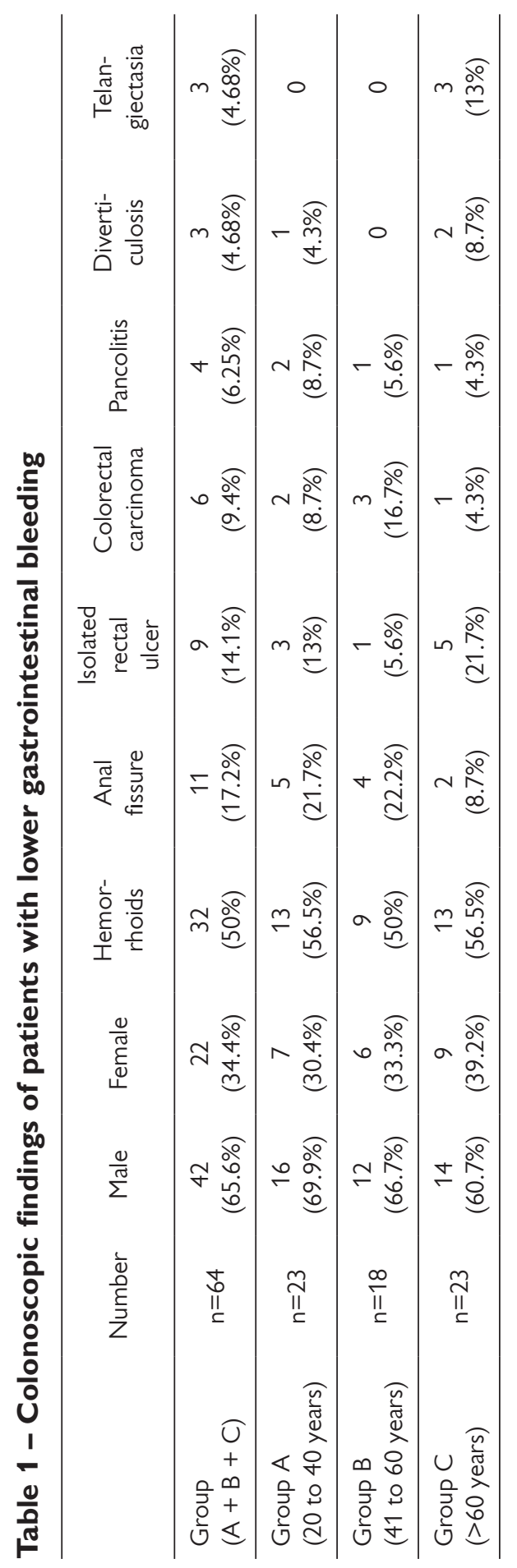




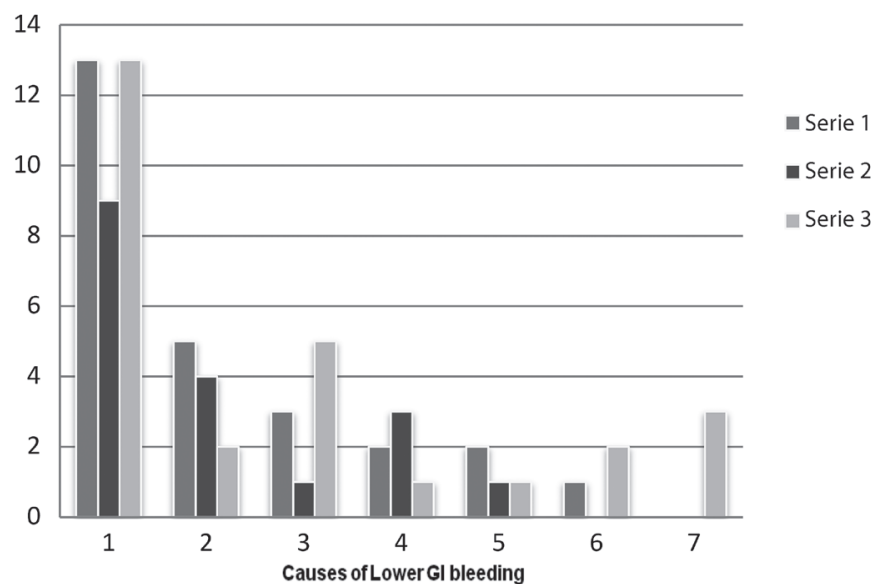

1: Hemorrhoids; 2: Anal fissure; 3 : Isolated rectal ulcer; 4: Colorectal carcinoma; 5 : Pan colitis; 6: Diverticulitis; 7 : Telangiectasia Figure 5 - Comparison of causes of lower gastrointestinal bleeding among different groups (series 1: group $A$, series 2: group $B$, series 3: group $C$ ).

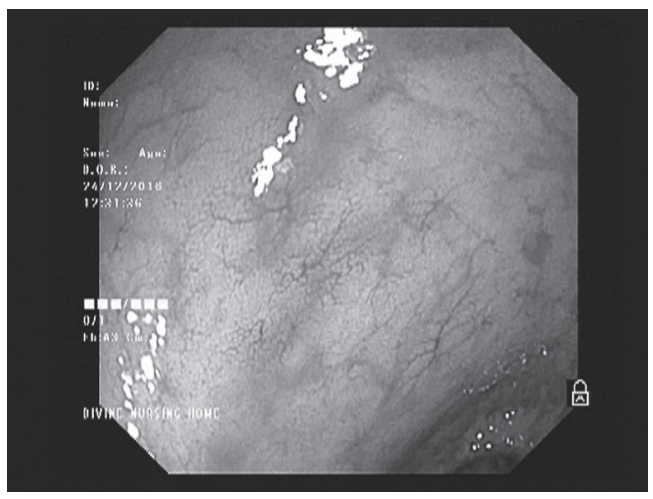

Figure 6 - Telangiectasia.

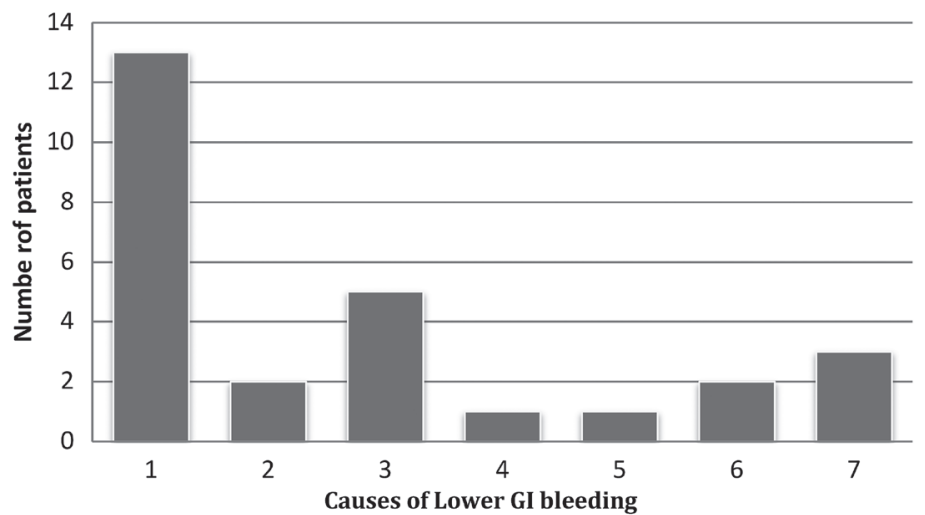

1: Hemorrhoids; 2: Anal fissure; 3: Isolated rectal ulcer; 4: Colorectal carcinoma; 5: Pan colitis; 6: Diverticulitis; 7: Telangiectasia

Figure 7 - Causes of lower gastrointestinal bleeding in group $C$ (more than 60 years) patients.

Paul J. 


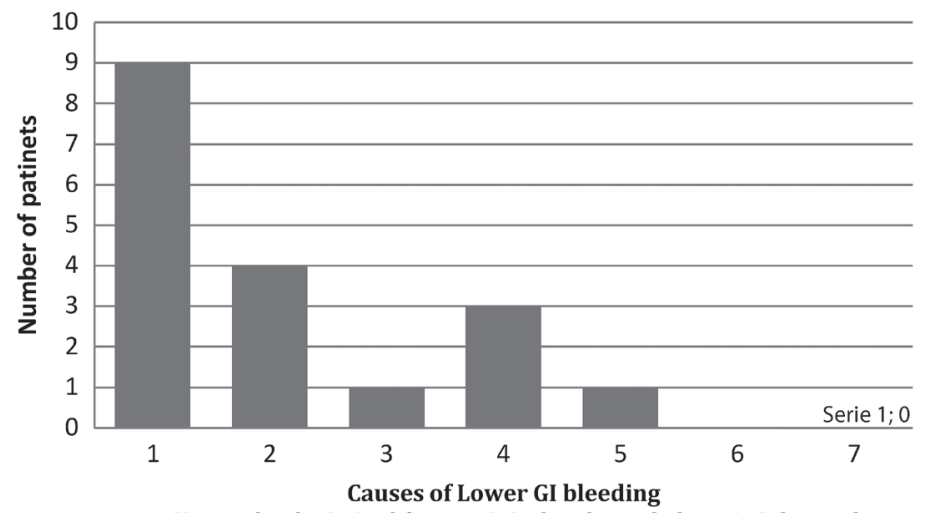

1: Hemorrhoids; 2: Anal fissure; 3: Isolated rectal ulcer; 4: Colorectal carcinoma; 5: Pan colitis; 6: Diverticulitis; 7: Telangiectasia

Figure 8 - Causes of lower gastrointestinal bleeding in group B (41 years to 60 years) patients.

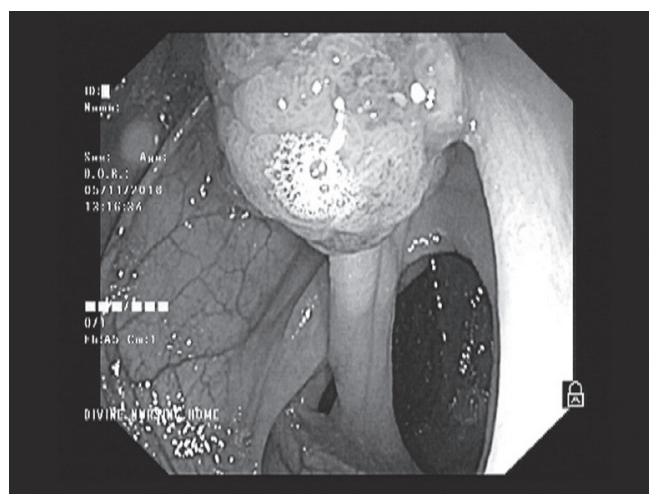

Figure 9 - Colon polyp

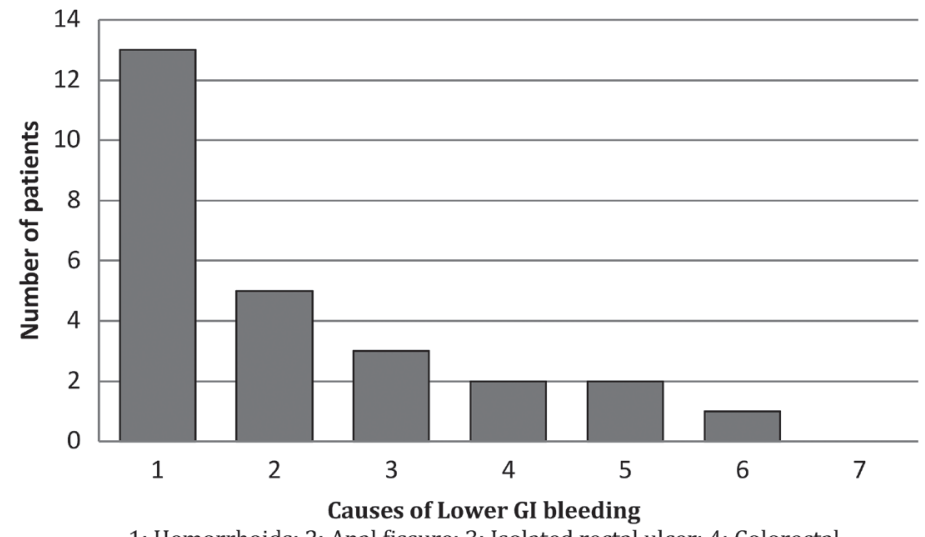

1: Hemorrhoids; 2: Anal fissure; 3: Isolated rectal ulcer; 4: Colorectal carcinoma; 5: Pan colitis; 6: Diverticulitis; 7: Telangiectasia

Figure 10 - Causes of lower gastrointestinal bleeding in group A (20 years to 40 years) patients. 
In group A (20 to 40 years), out of 23 patients, male and female patients were 16 (69.9\%) and 7 (30.4\%), respectively. Hemorrhoids was most common cause of LGIB $(n=13 ; 56.5 \%)$ in this group. Other causes of LGIB were anal fissure $(n=5 ; 21.7 \%)$, rectal ulcer $(n=3 ; 13 \%)$, colorectal growth $(n=2 ; 8.7 \%)$, colon polyp $(n=1 ; 4.3 \%)$ (Figure 9), pancolitis ( $n=2 ; 8.7 \%)$, diverticulae $(n=1 ; 4.3 \%)$ (Figure 10).

\section{Discussion}

The clinical course of LGIB can vary widely from occult bleeding to massive lifethreatening hemorrhage and even death but most patients who are having LGIB have favorable outcome, self-limited course and usually stopped spontaneously. Most of the LGIB patients think that the bleeding is from hemorrhoids and take some conservative measures, and some patients are worried and anxious about the malignancy until a diagnosis is reached. Causes of LGIB show marked geographic variation. Though studies from different parts of India (Dar et al., 2015; Camus et al., 2017) showed different common causes of LGIB, our study is supported by one study from South India (Badiger et al., 2017). Hajare and Kantamaneni (2018) in their study identified that hemorrhoids (48\%) followed by ulcerative colitis (24\%) were the most common colonoscopic findings in patients with LGIB. Another study from South India revealed that the most common causes of LGIB in patients older than 60 years (group C) were colorectal carcinoma, followed by colitis, hemorrhoids (Morkar and Hazare, 2017), but in our study, patients older than 60 years had hemorrhoids as most common cause of LGIB followed by rectal ulcer and telangiectasia, and colorectal carcinoma was more common in group B patients. One study from Europe (Fernández et al., 1996) indicated that more frequent colonoscopic findings were polyps and diverticulae in LGIB patients. In our study, in all age group hemorrhoids followed by anal fissure were the most common causes of LGIB.

One study from Iran (Khodadoostan et al., 2018) showed that most common causes of LGIB in patients younger than 50 years were hemorrhoids followed by adenoma and diverticulae but in our study, hemorrhoids followed by anal fissure and isolated rectal ulcer were the most common findings in colonoscopy.

Colorectal growth was more common in group $B$ than group $A$ and $C$ in our study and mean age of patient suffering from colorectal growth was $47 \pm 12.56$ years as seen in other study from India which showed mean age of 43 years (Sudarshan et al., 2013). Pancolitis was seen most commonly in group B as seen in other study (Quezada and Cross, 2012). Solitary rectal ulcerations were more common in group $C$ followed by group $A$ and $B$, and most of them have one or more of the following predisposing factors: constipation, straining during defecation and digital evacuation.

In our study, telangiectasia was seen in $4.68 \%$ of 64 patients and commonly seen in age more than 60 years (13\% of group C). Zia et al. (2008) supported our study by showing $1 \%$ colonoscopic finding of telangiectasia in their research. 
Main limitation of our study was small number of patients in study population; therefore large-scale study is required to validate the findings of this study.

\section{Conclusion}

Most common causes of LGIB in eastern part of India are hemorrhoids, anal fissure and isolated rectal ulcer. Male individuals are more commonly affected by LGIB. Colonic growth was seen more commonly in female patients. Any LGIB patient requires colonoscopy to identify the underlying cause of bleeding.

\section{References}

Alruzug, I. M., Aldarsouny, T. A., Semaan, T., AlMustafa, A. (2016) Lower gastrointestinal bleeding in Saudi patients: a retrospective longitudinal study. J. Gastrointest. Dig. Syst. 6, 410.

Badiger, R. H., Hajare, S., Kantamaneni, R., Kole, A., Deebanshu (2017) Etiological profile of patients presenting with lower gastrointestinal bleeding at tertiary care hospital at Belagavi: a cross sectional study. Int. J. Adv. Med. 4, 1429-1433.

Camus, M., Jensen, D. M., Ohning, G. V., Kovacs, T. O., Jutabha, R., Ghassemi, K. A., Machicado, G. A., Dulai, G. S., Jensen, M. E., Gornbein, J. A. (2017) Comparison of three risk scores to predict outcomes of severe lower gastrointestinal bleeding. J. Clin. Gastroenterol. 50, 52-58.

Dar, I. A., Dar, W. R., Khan, M. A., Kasana, B. A., Sofi, N. U., Hussain, M., Arshad, F., Wani, M. A., Latief, M., Sodhi, J. S. (2015) Etiology, clinical presentation, diagnosis and management of lower gastrointestinal bleed in a tertiary care hospital in India: A retroprospective study. J. Dig. Endosc. 6, 101-109.

de Souza e Benevides, I. B., dos Santos, C. H. M. (2016) Colonoscopy in the diagnosis of acute lower gastrointestinal bleeding. J. Coloproctol. 36, 185-188.

Diamantopoulou, G., Konstantakis, C., Kottorou, A., Skroubis, G., Theocharis, G., Theopistos, V., Triantos, C., Nikolopoulou, V., Thomopoulos, K. (2017) Acute lower gastrointestinal bleeding: Characteristics and clinical outcome of patients treated with an intensive protocol. Gastroenterology Res. 10, 352-358.

Dutta, G., Panda, M. (2008) An uncommon cause of lower gastrointestinal bleeding: a case report. Cases J. 1, 235.

Farrell, J. J., Friedman, L. S. (2005) Review article: The management of lower gastrointestinal bleeding. Aliment. Pharmacol. Ther. 21, 1281-1298.

Fernández, E., Linares, A., Alonso, J. L., Sotorrio, N. G., de la Vega, J., Artimez, M. L., Giganto, F., Rodríguez, M., Rodrigo, L. (1996) Colonoscopic findings in patients with lower gastrointestinal bleeding send to a hospital for their study. Value of clinical data in predicting normal or pathological findings. Rev. Esp. Enferm. Dig. 88, 16-25.

Ghassemi, K. A., Jensen, D. M. (2013) Lower Gl bleeding: Epidemiology and management. Curr. Gastroenterol. Rep. 15, 333.

Hajare, S., Kantamaneni, R. (2018) Etiological profile of patients with lower gastrointestinal bleeding: A 1-year cross-sectional study. Arch. Med. Health Sci. 6, 300-302.

Khodadoostan, M., Shavakhi, A., Padidarnia, R., Shavakhi, A., Ahmadian, M. (2018) Full colonoscopy in patients under 50 years old with lower gastrointestinal bleeding. J. Res. Med. Sci. 23, 45.

Morkar, D. N., Hazare, S. (2017) Spectrum of the causes of lower gastrointestinal bleeding in geriatric patients in tertiary care hospital. J. Sci. Soc. 44, 148-151.

Oakland, K., Chadwick, G., East, J. E., Guy, R., Humphries, A., Jairath, V., McPherson, S., Metzner, M., Morris, A. J., Murphy, M. F., Tham, T., Uberoi, R., Veitch, A. M., Wheeler, J., Regan, C., Hoare, J. (2019) 
Diagnosis and management of acute lower gastrointestinal bleeding: Guidelines from the British Society of Gastroenterology. Gut 68, 776-789.

Quezada, S. M., Cross, R. K. (2012) Association of age at diagnosis and ulcerative colitis phenotype. Dig. Dis. Sci. 57, 2402-2407.

Sudarshan, V., Hussain, N., Gahine, R., Mourya, J. (2013) Colorectal cancer in young adults in a tertiary care hospital in Chhattisgarh, Raipur. Indian J. Cancer 50, 337-340.

Zia, N., Hussain, T., Salamat, A., Mirza, S., Hassan, F., Waqar A. (2008) Diagnostic evaluation of patients presenting with bleeding per rectum by colonoscopy. J. Ayub Med. Coll. Abbottabad 20, 73-76. 